\title{
JPFSM: Short Review Article \\ The important role of the neuromuscular junction in maintaining muscle mass and strength
}

\author{
Shuuichi Mori*, Katsuo Koshi and Kazuhiro Shigemoto \\ Department of Geriatric Medicine, Tokyo Metropolitan Institute of Gerontology, 35-2 Sakae-cho, Itabashi-ku, Tokyo 173-0015, Japan
}

Received: December 11, 2013 / Accepted: December 19, 2013

\begin{abstract}
Sarcopenia is an age-related decline of skeletal muscle mass and function, leading to reduced physical ability and difficulties in carrying out day-to-day tasks; it has become a major health concern in our aging society. The exact etiology of sarcopenia is not completely understood, but recent studies have focused on age-associated changes in the neuromuscular junction (NMJ). Using an animal model of myasthenia gravis with antibodies against musclespecific kinase (MuSK), a disorder of the NMJ characterized by muscle weakness, we investigated the role of the NMJ in the pathogenesis of sarcopenia. Our studies indicate that the structure and function of the NMJ is important for maintaining muscle mass and strength, which suggests that the malfunction of the NMJ plays a role, at least in part, in the onset of sarcopenia. These findings suggest that the NMJ will become an important therapeutic target for sarcopenia in the future.
\end{abstract}

Keywords : neuromuscular junction, sarcopenia, myasthenia gravis, muscle-specific kinase (MuSK)

\section{Introduction}

Aging gradually causes a decrease in physical ability and the ease of carrying out daily tasks; quality of life is also usually impaired. The fundamental cause of these problems is an age-associated decline in skeletal muscle mass and function, a condition known as sarcopenia ${ }^{1,2}$. With our increasingly aging society, prevention and treatment of sarcopenia is essential in order to reduce the risk of morbidity and mortality in the elderly, as well as alleviate the financial burden on family members. Research has focused on improving our understanding of the etiology of sarcopenia, which should lead to new methods being devised for preventing and treating this disability. The etiology of sarcopenia is thought to be a multi-factorial process that involves both intrinsic and extrinsic factors. Various physical mechanisms have been postulated to contribute to sarcopenia, including decreased capacity of motoneurons to innervate muscle fibers; a decline in satellite cell activation and proliferation; oxidative damage; and hormone deficiencies associated with aging. In this review, we present recent data on proposed mechanisms that contribute to the onset of sarcopenia, focusing on the role of the neuromuscular junction (NMJ).

\section{Structure of the NMJ}

The NMJ is a joint between motoneurons and skeletal muscle, and is composed of three regions: the presyn-

\footnotetext{
*Correspondence: shuuichi@tmig.or.jp
}

aptic part (the motor nerve terminal), the synaptic cleft, and the postsynaptic part (the muscle membrane; Fig. 1). The myelin sheath surrounding presynaptic motor axons, extended from motoneurons in the anterior horn of the spinal cord, ends in proximity to the muscle fibers; the axons then terminate in a shallow depression of the muscle membrane. The motor nerve terminals contain a large number of synaptic vesicles, each of which stores approximately 5,000 to 10,000 molecules of the neurotransmitter acetylcholine (ACh). When an action potential arrives at the terminal, an increase in the local concentration of free $\mathrm{Ca}^{2+}$ (through voltage-gated $\mathrm{Ca}^{2+}$ channels) facilitates the

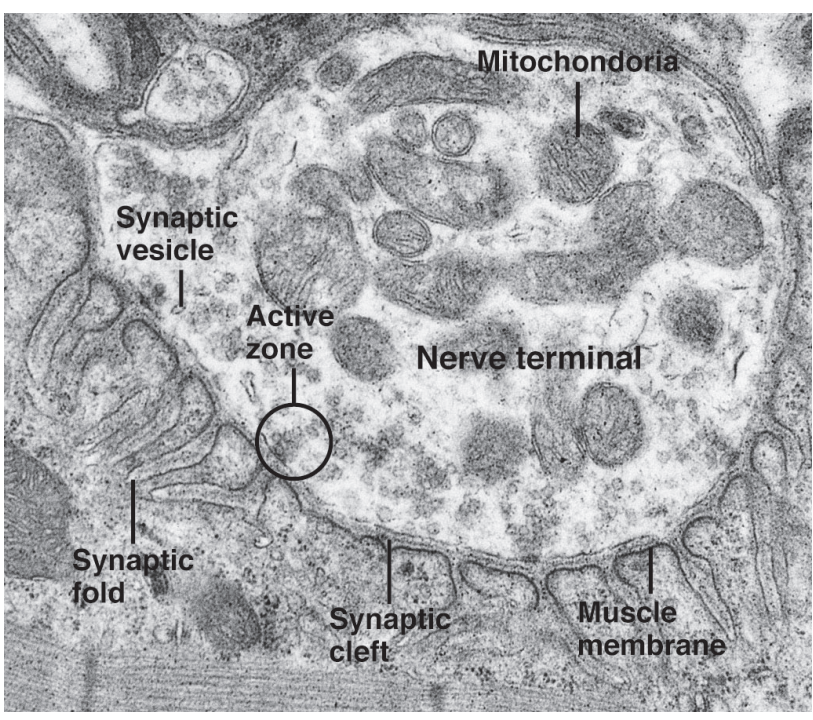

Fig. 1 Electron micrograph of a mouse neuromuscular junction. 
fusion of synaptic vesicles with the presynaptic membrane, at a site called the 'active zone'. ACh is then released from overlying active zones in the nerve terminals and binds to ACh receptors (AChRs) in the muscle membrane. This causes ligand-gated ion channels to open and depolarizes the muscle membrane, which generates an action potential that stimulates muscle contraction. In particular, the postsynaptic membrane has a large number of deep invaginations, termed synaptic folds, which increase the surface area of the membrane exposed to the nerve terminal. Moreover, AChRs are highly concentrated at the crests of postjunctional folds ( $>10,000$ receptors per $\left.\mu \mathrm{m}^{2}\right)$, compared to the troughs of those folds and in extrasynaptic regions, where the density of receptors is $<10$ sites per $\mu \mathrm{m}^{2}{ }^{3,4)}$. As described above, the NMJ is a specialized structure with a number of properties that ensure effective neuromuscular transmission.

\section{Age-associated changes of the NMJ}

Morphological changes of the skeletal muscle found in patients with sarcopenia, include a reduced number of muscle fibers, greater heterogeneity of fiber thickness, the presence of angular fibers, an increased number of centralized myonuclei, fiber-type grouping, and a preponderance of type I fibers ${ }^{5}$. Several lines of evidence from both animal and human studies have suggested that these changes mainly result from degeneration of motoneurons and denervation of skeletal muscle fibers ${ }^{2,6,7)}$. A preferential denervation of type II fibers and reinnervation by axonal sprouting from motoneurons innervating type I fibers, leads to remodeling of the motor units, which results in fiber-type grouping and preponderance of type I fibers. However, in the condition in which the denervation outpaces the reinnervation, muscle fibers, which are not reinnervated, degenerate and disappear. This atrophy of the muscle fibers ultimately leads to the loss of skeletal muscle mass, which contributes to the decline in muscle strength seen in sarcopenia. Interestingly, it has been suggested that age-associated changes in the structure of the NMJ precede the denervation of muscle fibers ${ }^{6,8)}$. Anatomical studies in laboratory animals have shown that, in aged muscles, some presynaptic nerves exhibit terminal sprouts, and that postsynaptic AChR clusters are often fragmented into lots of small islands instead of a typical pretzel-like structure; however, these structures are rarely observed at NMJs in young adult muscles ${ }^{6-10)}$. In addition, electron microscopic study has shown that labyrinthine synaptic gutters observed at young adult NMJs become simplified into a number of cup-like depressions at aged $\mathrm{NMJs}^{11)}$. On the presynaptic side, it has been shown that the number of active zones and the expression level of Bassoon, a scaffold protein essential for assembly of the active zone, was significantly reduced at the nerve terminals of NMJs in aged mice ${ }^{12,13)}$. The living body is intrinsically equipped with homeostatic mechanisms; however, these changes suggest that aging impairs the capability of the NMJ to maintain its structural integrity. As described above, the complicated structure of the NMJ is essential for effective neuromuscular transmission, and it is thought that the age-associated distortion of the NMJ could reduce the efficiency of neuromuscular transmission, and hence contribute to the impairment of muscle contraction. It has been reported that the 'safety factor', a measure of the ability of neuromuscular transmission to remain effective under various physiological conditions, declines at NMJs of the diaphragm in aged rats ${ }^{14)}$. Taken together, it is possible that failure of the mechanism needed to maintain the $\mathrm{NMJ}$, plays a role in the onset of sarcopenia.

\section{Role of MuSK in maintaining the structure and func- tion of the NMJ}

MuSK (muscle-specific kinase) is a receptor-tyrosine kinase which is concentrated at the peaks of synaptic folds at the NMJ alongside AChRs. MuSK is activated by agrin, a nerve-derived heparan sulfate proteoglycan, through the LDL receptor-related protein 4 (LRP4). In addition to agrin, MuSK activation also requires interaction with Dok-7, a cytoplasmic adaptor protein. The dual activation of MuSK results in the interaction of several downstream molecules, leading to clustering of $\mathrm{AChRs}^{15)}$. MuSK-deficient mice display devastating defects in both pre- and post- synaptic differentiation and die at birth because they cannot breathe. In these mice, motor nerves grow excessively and fail to form terminal arbors, and no AChR clusters are present on myofibers opposite ingrowing nerve terminals. These data show that MuSK is essential for the formation of NMJs at the embryonic stage ${ }^{16)}$.

Autoantibodies against MuSK have been found in a proportion of patients with myasthenia gravis (MG), a disorder of the NMJ characterized by ptosis, fatigue and muscle weakness ${ }^{17)}$. MG is an autoimmune disease caused by an immunological response against specific proteins on postsynaptic membranes; experimental autoimmune $\mathrm{MG}$ (EAMG) is an animal model used to investigate the pathogenic mechanisms of MG by replicating the symptoms of human disease. We previously developed a model of EAMG caused by anti-MuSK antibodies (MuSK-EAMG) by injecting rabbits and complement-deficient mice with recombinant soluble MuSK protein ${ }^{18-20)}$. EAMG using complement-deficient mice eliminated the possible effect of immune complex-mediated damage to tissue at the onset of MG because of failure in activation of the complement cascade, providing direct evidence that disruption of MuSK by autoantibodies causes MG. Mice affected with MuSK-EAMG exhibited marked muscle weakness, muscle fatigue caused by impairment of neuromuscular transmission, and muscle atrophy. Morphological analysis indicated significant loss of AChR expression, retraction of nerve terminals opposite AChR clusters, axon sprouting (with or without the remnant of nerve terminals), and 
a reduction of synaptic folds at NMJs. In addition to the morphological defects, functional defects at the NMJ were also observed. Electrophysiological assessment of the efficiency of neuromuscular transmission ex vivo indicated that lower levels of ACh were being released from presynaptic nerve terminals in these mice. These observations demonstrate that inhibition of MuSK with autoantibodies disturbs both pre- and post- synaptic structure and function at the NMJ, despite the fact that MuSK is only expressed at postsynaptic membranes, and suggest that MuSK acts via retrograde signals from postsynaptic sites to maintain presynaptic structure and function. Taken together, our studies illustrate that MuSK plays an important bidirectional role in maintaining the structure and function of the mature NMJ, and that blocking MuSK action leads to a breakdown in NMJ function and causes MG. Although MG and sarcopenia have a different initial cause, it is possible that they share a common fundamental mechanism, i.e., disruption of NMJ structure and function leading to muscle weakness and atrophy, which is observed in both diseases ${ }^{21)}$. Therefore, we believe that studying MG, caused by inhibition of MuSK, is a useful method for trying to resolve the pathogenic mechanisms of sarcopenia.

\section{Relationship between MuSK and sarcopenia}

To date, studies have implied an indirect relationship between MuSK and sarcopenia. Agrin, an activator of MuSK, is degraded by neurotrypsin, a neuronal serine protease. Young adult mice with motoneuron-specific neurotrypsin overexpression in which degradation of agrin is enhanced, exhibit structural changes of the NMJ, including fragmentation of AChR clusters, a reduction in the number of muscle fibers, the presence of centralized myonuclei, and an increased proportion of type I fibers, all of which are associated with sarcopenia ${ }^{22)}$. These results are consistent with the notion that decreased functionality of the NMJ plays a role in the onset of sarcopenia, and suggest that deactivation of MuSK, by the removal of agrin from the NMJ, is part of the pathogenic mechanism. Interestingly, both neurotrypsin-deficient and neurotrypsin-resistant agrin-overexpressing mice, in which levels of agrin are elevated, develop sarcopenia at old age, suggesting that multiple mechanisms contribute to the onset of sarcopenia. Neurotrypsin-overexpressing mice could therefore represent another model for studying the pathology of sarcopenia in addition to MuSK-EAMG mice.

\section{Future perspective on research into sarcopenia}

Given the hypothesis that decreased functionality of the NMJ results in the onset of sarcopenia, it is feasible to suggest that increasing the stability of the NMJ could prevent or delay the disease course. Animal studies have shown that the NMJ exhibits considerable structural and functional plasticity in response to altered levels of neuromuscular activity such as through exercise training or disuse $^{23)}$. Interestingly, in aged mice, one month of voluntary exercise on running wheels was reported to attenuate and partially reverse age-related changes in NMJ structures that had already occurred ${ }^{10)}$. Furthermore, it was shown that isometric strength training for two months in aged rats could lessen the decrease in Bassoon at the $\mathrm{NMJ}^{13)}$. These observations highlight the beneficial effect of physical activity. Although the detailed mechanism is not yet known, increased muscle activity through exercise could potentiate expression and release of muscle-derived trophic factors that improve the functionality of the NMJ in an autocrine and/or paracrine fashion. It has recently been reported that skeletal muscle cells release a number of humoral factors termed 'myokines" ${ }^{\text {'2) }}$. Further identification of novel myokines activated by exercise is needed, and the effect of myokines on NMJ plasticity should be investigated.

Another approach in the search for unraveling a potential mechanism for maintaining NMJ structure and function is to develop biomarkers for sarcopenia. Recently, an agrin fragment cleaved by neurotrypsin in blood has been identified as a marker for the diagnosis of sarcopenia in Europe ${ }^{25,26)}$. Early detection of sarcopenia using such biomarkers is important for initiating treatment early in patients and for monitoring the efficacy of treatment continuously. In conclusion, the NMJ will become an important therapeutic target for sarcopenia in the future.

\section{Acknowledgments}

This work was supported by a Grant-in-Aid for Young Scientists (B) (25860734) from the Ministry of Education, Science, and Culture, Japan.

\section{References}

1) Roubenoff R and Hughes VA. 2000. Sarcopenia: current concepts. J Gerontol A Biol Sci Med Sci 55: M716-M724.

2) Doherty TJ. 2003. Invited review: Aging and sarcopenia. $J$ Appl Physiol 95: 1717-1727.

3) Hartzell HC and Fambrough DM. 1973. Acetycholine receptor production and incorporation into membranes of developing muscle fibers. Dev Biol 30: 153-165.

4) Cohen SA and Fischbach GD. 1977. Clusters of acetylcholine receptors located at identified nerve-muscle synapses in vitro. Dev Biol 59: 24-38.

5) Lexell J, Taylor CC and Sjostrom M. 1988. What is the cause of the ageing atrophy? Total number, size and proportion of different fiber types studied in whole vastus lateralis muscle from 15- to 83-year-old men. J Neurol Sci 84: 275-294.

6) Deschenes MR, Roby MA, Eason MK and Harris MB. 2010. Remodeling of the neuromuscular junction precedes sarcopenia related alterations in myofibers. Exp Gerontol 45: 389393. 
7) Jang YC and Van Remmen H. 2011. Age-associated alterations of the neuromuscular junction. Exp Gerontol 46: 193198.

8) Li Y, Lee Y and Thompson WJ. 2011. Changes in aging mouse neuromuscular junctions are explained by degeneration and regeneration of muscle fiber segments at the synapse. J Neurosci 31: 14910-14919.

9) Balice-Gordon RJ. 1997. Age-related changes in neuromuscular innervation. Muscle Nerve Suppl 5: S83-S87.

10) Valdez G, Tapia JC, Kang H, Clemenson GD, Jr., Gage FH, Lichtman JW and Sanes JR. 2010. Attenuation of age-related changes in mouse neuromuscular synapses by caloric restriction and exercise. Proc Natl Acad Sci USA 107: 1486314868.

11) Ezaki T, Oki S, Matsuda Y and Desaki J. 2000. Age changes of neuromuscular junctions in the extensor digitorum longus muscle of spontaneous thymoma BUF/Mna rats. A scanning and transmission electron microscopic study. Virchows Arch 437: 388-395.

12) Chen J, Mizushige $T$ and Nishimune H. 2012. Active zone density is conserved during synaptic growth but impaired in aged mice. J Comp Neurol 520: 434-452.

13) Nishimune H, Numata T, Chen J, Aoki Y, Wang Y, Starr MP, Mori Y and Stanford JA. 2012. Active zone protein Bassoon co-localizes with presynaptic calcium channel, modifies channel function, and recovers from aging related loss by exercise. PLoS One 7: e38029.

14) Kelly SS. 1978. The effect of age on neuromuscular transmission. J Physiol 274: 51-62.

15) Wu H, Xiong WC and Mei L. 2010. To build a synapse: signaling pathways in neuromuscular junction assembly. Development 137: 1017-1033.

16) DeChiara TM, Bowen DC, Valenzuela DM, Simmons MV, Poueymirou WT, Thomas S, Kinetz E, Compton DL, Rojas E, Park JS, Smith C, DiStefano PS, Glass DJ, Burden SJ and Yancopoulos GD. 1996. The receptor tyrosine kinase MuSK is required for neuromuscular junction formation in vivo. Cell 85: 501-512.

17) Hoch W, McConville J, Helms S, Newsom-Davis J, Melms A and Vincent A. 2001. Auto-antibodies to the receptor tyrosine kinase MuSK in patients with myasthenia gravis without acetylcholine receptor antibodies. Nat Med 7: 365-368.

18) Shigemoto K, Kubo S, Maruyama N, Hato N, Yamada H, Jie C, Kobayashi N, Mominoki K, Abe Y, Ueda N and Matsuda S. 2006. Induction of myasthenia by immunization against muscle-specific kinase. J Clin Invest 116: 1016-1024.

19) Mori S, Kubo S, Akiyoshi T, Yamada S, Miyazaki T, Hotta H, Desaki J, Kishi M, Konishi T, Nishino Y, Miyazawa A, Maruyama N and Shigemoto K. 2012. Antibodies against muscle-specific kinase impair both presynaptic and postsynaptic functions in a murine model of myasthenia gravis. Am J Pathol 180: 798-810.

20) Mori S, Kishi M, Kubo S, Akiyoshi T, Yamada S, Miyazaki T, Konishi T, Maruyama N and Shigemoto K. 2012. 3,4-Diaminopyridine improves neuromuscular transmission in a MuSK antibody-induced mouse model of myasthenia gravis. $J \mathrm{Neu}$ roimmunol 245: 75-78.

21) Shigemoto K, Kubo S, Mori S, Yamada S, Akiyoshi T and Miyazaki T. 2010. Muscle weakness and neuromuscular junctions in aging and disease. Geriatr Gerontol Int 10 Suppl 1: S137-S147.

22) Butikofer L, Zurlinden A, Bolliger MF, Kunz B and Sonderegger P. 2011. Destabilization of the neuromuscular junction by proteolytic cleavage of agrin results in precocious sarcopenia. FASEB J 25: 4378-4393.

23) Wilson MH and Deschenes MR. 2005. The neuromuscular junction: anatomical features and adaptations to various forms of increased, or decreased neuromuscular activity. Int J Neurosci 115: 803-828.

24) Manabe Y, Miyatake S and Takagi M. 2012. Myokines: Do they really exist? J Phys Fitness and Sports Med 1: 51-58.

25) Drey M, Sieber CC, Bauer JM, Uter W, Dahinden P, Fariello RG and Vrijbloed JW. 2013. C-terminal Agrin Fragment as a potential marker for sarcopenia caused by degeneration of the neuromuscular junction. Exp Gerontol 48: 76-80.

26) Hettwer S, Dahinden P, Kucsera S, Farina C, Ahmed S, Fariello R, Drey M, Sieber CC and Vrijbloed JW. 2013. Elevated levels of a C-terminal agrin fragment identifies a new subset of sarcopenia patients. Exp Gerontol 48: 69-75. 